\title{
A disk-type dose imaging detector based on blue and orange RPL in Ag-activated phosphate glass for 2D and 3D dose imaging applications
}

\author{
Toshio Kurobori ${ }^{\mathrm{a}, *}$, Akihiro Takemura ${ }^{\mathrm{b}}$, Yuka Miyamoto ${ }^{\mathrm{c}}$, Daisuke Maki ${ }^{\mathrm{c}}$, Yasuhiro \\ Koguchi ${ }^{\mathrm{c}}$, Nobuhiro Takeuchi ${ }^{\mathrm{c}}$, Takayoshi Yamamoto ${ }^{\mathrm{c}}$, Yao Qiang Chen ${ }^{\mathrm{d}}$ \\ ${ }^{a}$ Graduate School of Natural Science \& Technology, Kanazawa University, Kanazawa 920-1192, Japan \\ ${ }^{\mathrm{b}}$ Graduate School of Medical Science, Kanazawa University, Kanazawa 920-0942, Japan \\ ${ }^{\mathrm{c}}$ Oarai Research Center, Chiyoda Technol Corpration., Oarai 311-1313, Japan \\ ${ }^{\mathrm{d}}$ China Techwin Co., Ltd, Dongguan, Guangdong 523467, China
}

\begin{abstract}
A disk-type two- and three-dimensional (2D, 3D) X-ray dose imaging detector was developed based on the radiation-induced silver (Ag)-related species in Ag-activated phosphate glass. This luminescent detector is based on the blue and orange radiophotoluminescence (RPL) phenomena. A comparative study of the steady- and transient-state optical properties of blue and orange RPL, such as the time-resolved luminescence spectra and the RPL decay curves, was performed. In addition, the combination of a confocal optical detection system with a transparent glass detector enables 3D reconstruction through the stacking of dose images collected at different depths within the material. The capabilities of this detector for $2 \mathrm{D}$ and $3 \mathrm{D}$ dose imaging applications are discussed.
\end{abstract}

Keywords: Ag-activated phosphate glass, X-ray imaging, Fluorescent detection Radiophotoluminescence (RPL), Passive dosimeter

*Corresponding author. Tel.: +81-76-264-5478; fax: +81-76-234-4132. E-mail address: kurobori@staff.kanazawa-u.ac.jp (T. Kurobori). 


\section{Introduction}

Dosimeters consisting of silver (Ag)-activated phosphate glass, which is most commonly known as radiophotoluminescent material, are recognized to possess several desirable characteristics, such as non-destructive readout capability, long-term stability against fading, a wide dynamic range and uniformity/batch homogeneity (Yokota and Imagawa, 1966; Perry, 1987; Yamamoto et al., 2011).

Recently, a novel disk-type two-dimensional (2D) imaging detector with the aforementioned superior characteristics of radiophotoluminescent dosimeters has been proposed and demonstrated (Kurobori and Nakamura, 2012) for the first time. In addition, a comparative study has been performed of the 2D dose images acquired using Ag-activated phosphate glass based on the orange radiophotoluminescence (RPL) of atomic-scale Ag-related species and those acquired using LiF thin films based on the photoluminescence (PL) of F-aggregate colour centres (CCs) (Montereali et al., 1991), and the performances of the two techniques have been evaluated (Kurobori et al., 2014a). These disk-type detectors, with a diameter of $80 \mathrm{~mm}$ and a thickness of $1 \mathrm{~mm}$, were rotated at a rate of $2400 \mathrm{rpm}(40 \mathrm{~Hz})$. A detailed description of the optical and dosimetric properties of Ag-related defects in Ag-activated phosphate glass has been published elsewhere (Kurobori et al., 2010; Zheng and Kurobori, 2011).

In this paper, the basic operating principles of detectors are described first based on the blue and orange RPL Ag-activated phosphate glass. Second, a room-temperature (RT) study of the steady- and transient-state optical properties of the blue and orange RPL of such detectors, including the time-resolved luminescence spectra and the decay curves, is 
presented to evaluate and understand accurate 3D dose imaging. Specifically, certain advantages of using the blue RPL instead of the typically used orange RPL are demonstrated and discussed. Finally, it is demonstrated that the use of a confocal detection system and a transparent glass detector allows one to reconstruct a three-dimensional (3D) dose distribution by combining images collected at different depths, from near the surface to a depth of $\approx 400 \mu \mathrm{m}$.

\section{Principles of the detector}

The basic principles for image acquisition and processing using Ag-activated phosphate glass can be divided into three separate steps, i.e., the recording, reading and erasing processes, as illustrated in Fig. 1. In step 1, the RPL glass detector is exposed to ionizing radiation through a subject. Before exposure, the Ag-activated glass consists of a substrate that contains positively charged silver ions $\left(\mathrm{Ag}^{+}\right)$and negatively charged phosphate ions $\left(\mathrm{PO}_{4}{ }^{3-}\right)$. After exposure, electron-trapped and hole-trapped silver centres are produced within the glass; however, the irradiated glass remains transparent, and no image can be observed in the visible wavelength range. For the visualization of a dose image, the glass is exposed to ultraviolet (UV) light (typically a black light); intense, orange-coloured emission can then be observed. This phenomenon is generally known as RPL (Perry, 1987). The irradiated glass must be properly read out to reconstruct a dose image. In step 2, before readout, the glass plate must undergo a heat treatment ('pre-heating', $\mathrm{PH}$ ) at $100{ }^{\circ} \mathrm{C}$ for 10 min to complete the 'build-up' kinetics of the Ag-related species, specifically, $\mathrm{Ag}^{2+}$ hole-trapped silver centres. After the PH step, the glass detector is exposed to a UV laser 
for readout, and the RPL signal is detected by a photomultiplier tube (PMT) and digitized using an analogue-to-digital (A/D) converter. Then, the dose distribution is reconstructed using a personal computer (PC). In step 3, the residual radiation image of the glass detector can be erased by annealing the detector for $10 \mathrm{~min}$ at $360{ }^{\circ} \mathrm{C}$, allowing it to be used repeatedly.

\section{Experimental procedure}

\subsection{Ag-activated phosphate glass}

A piece of commercially available Ag-activated phosphate glass was used as a $2 \mathrm{D}$ and 3D RPL detector. Although the weight composition of the detector used in this study was the same as that of the FD-7 (Asahi Techno Glass), i.e., 31.55\% P, 51.16\% O, 6.12\% Al, $11.00 \% \mathrm{Na}$ and $0.17 \% \mathrm{Ag}$, the size and shape were different; the detector was a disk-type plate with a diameter of $100 \mathrm{~mm}$ and a thickness of $1 \mathrm{~mm}$.

\subsection{X-ray irradiation}

The samples were coloured via irradiation from an X-ray unit with a copper target operating at $30 \mathrm{kV}$ and $20 \mathrm{~mA}$ at RT. The absorbed doses to the samples ranged from 0.1 to $10 \mathrm{~Gy}$. The dose rate from the X-ray unit was calibrated using a commercially available Ag-activated phosphate glass dosimeter.

\subsection{Steady-state optical properties}


Optical absorption was determined at RT using a Hitachi U-3900H UV-Vis spectrophotometer. The RPL spectra were obtained using a Hitachi F-2500 fluorescence spectrophotometer. For all optical measurements, the samples were cut into rectangular plates of a suitable size of approximately $10 \times 7 \times 1 \mathrm{~mm}^{3}$ from the original glass dosimeter plate.

\subsection{Transient-state optical properties}

The time-resolved RPL spectra and decay curves for the blue and orange RPL were acquired using a combination of a high-repetition-rate Q-switched laser (Explorer One 349, Spectra Physics) at $349 \mathrm{~nm}$ and a photonic multichannel analyzer (PMA-12, Hamamatsu Photonics), as shown in Fig. 2(a). The pulse duration of the laser was less than 5 ns full width at half maximum (FWHM) at a repetition rate of $1 \mathrm{kHz}$ for a pulse energy of $120 \mu \mathrm{J}$.

The area of the beam on the glass sample was $3 \times 0.14 \mathrm{~mm}^{2}$, and the fluence was 48 $\mu \mathrm{J} / \mathrm{mm}^{2}(20 \mu \mathrm{J} /$ pulse $)$. The time-resolved RPL spectra were measured in the wavelength range from 200 to $800 \mathrm{~nm}$ with a $1 \mathrm{~ns}$ step and a $10 \mathrm{~ns}$ gate time using a PMA-12 equipped with an image intensifier. Therefore, the total temporal resolution was approximately 10 ns.

\section{5. $2 D$ and $3 D$ imaging readout system}

A schematic view of the experimental arrangement employed to measure the $2 \mathrm{D}$ and $3 \mathrm{D}$ dose distributions is shown in Fig. 2(b). Collimated light at $371 \mathrm{~nm}$ from a cw laser diode (LuxX 371-70, Omicron GmbH) was used as an excitation source. For reading, a low-level power between 10 and $20 \mathrm{~mW}$ was used for stimulating the blue and orange RPL. The 
laser beam was expanded and reflected with a dichroic beam splitter (B/S) (\#86-330, Edmund Optics) and focused in the vicinity of the surface for the disk-type detector by a Nikon LU Plan Fluor objective lens $(100 \times, \mathrm{NA}=0.90)$ while reading out the image. The objective lens was simultaneously used for both laser excitation and emission collection. Each detector was attached to a spindle on a linear translation stage, which was rotated at a rate of $2400 \mathrm{rpm}$ and controlled to translate the laser beam spot from the outer disk to the inner disk in the radial direction with a pitch of $20 \mu \mathrm{m}$. Additional optical filters, including band-pass for simulating UV light (BP)(\#86-736, 375 $\pm 5 \mathrm{~nm}$, Edmund Optics), long-pass (LP) (\#62-981, Edmund Optics), BP for the orange RPL (\#84-797, 600 $\pm 25 \mathrm{~nm}$, Edmund Optics) and BP for the blue RPL (\#86-962, 475 25 nm, Edmund Optics), were inserted to reject the reflected emission and residual stimulating laser light, respectively. Only the signal was detected by the photomultiplier tube (PMT) (\#H10720-20, Hamamatsu Photonics), which then digitized the signal using a $500-\mathrm{kHz}, 16-$ bit analogue-to-digital (A/D) converter. The dose distribution was reconstructed by a personal computer. The total readout and reconstructed times were one to five min, depending on the track pitch (10 to $100 \mu \mathrm{m})$ and the number of tracks.

\section{Results and discussion}

Fig. 3(a) presents the RPL spectra of the Ag-activated phosphate glass with a thickness of $1 \mathrm{~mm}$ before (non-irradiated) and after X-ray irradiation for absorbed doses from 0.1 to $10 \mathrm{~Gy}$. Each Ag-activated glass plate was heated to $100{ }^{\circ} \mathrm{C}$ for $10 \mathrm{~min}$ to suppress the 'build-up' kinetics after X-ray irradiation, as shown in Fig. 1. The shapes of 
the absorption spectra were attributed to the superposition of a number of individual absorption bands corresponding to Ag atoms, charged Ag-aggregate CCs, and neutral Ag molecular clusters and phosphorous- and oxygen-related species in the range from 250 to $700 \mathrm{~nm}$ (not shown here), as previously reported (Kurobori et al., 2010; Zheng and Kurobori, 2011). The orange RPL peaks at $560 \mathrm{~nm}$ and is attributed to the $\mathrm{Ag}^{2+}$ centres; there is also another band that peaks at $450 \mathrm{~nm}$, which is known as the blue RPL and is attributed to the $\mathrm{Ag}^{0}$ centres. Blue and orange RPL are simultaneously emitted at an excitation wavelength of $349 \mathrm{~nm}$ for the measurement of optical properties or $371 \mathrm{~nm}$ for the measurement of $2 \mathrm{D}$ and $3 \mathrm{D}$ dose imaging because of the superposition of the characteristic absorption peaks of the blue and orange RPL, which occur at 345 and 310 $\mathrm{nm}$, respectively. Note that the dosimetric relationship between the intensity response of the blue and orange RPL and the absorbed X-ray doses from 0.1 to 10 Gy exhibits good dose linearity.

The time-resolved RPL spectra of the Ag-activated phosphate glass irradiated with 693,000 pulses are presented in Fig. 3(b) for several pulse delays after the $349 \mathrm{~nm}$ excitation pulse. For a delay of less than a zero delay, the luminescence signal is indistinguishable from the background. After a zero delay, a broadband spectrum peaking at 450 and $650 \mathrm{~nm}$ is observed. Note that the RPL spectra corresponding to the transient-state time scale are completely different from those corresponding to the steady-state time scale that are presented in Fig. 3(a), and note that the peak wavelength for the orange RPL was markedly shifted to a longer wavelength, from 560 to $650 \mathrm{~nm}$, upon UV nanosecond laser excitation. At further delays from 4 to $50 \mathrm{~ns}$, the intensity of the blue RPL band decreased rapidly while the intensity of the orange RPL band progressively decreases. Plotting the intensity of the bands at 450 and $650 \mathrm{~nm}$ versus the delay time after 
the pulse, as shown in Fig. 3(c), reveals the luminescence decay lifetimes. The observed lifetime values are compatible with those measured previously (Kurobori et al., 2014b) using a fluorescence lifetime spectrometer for X-ray irradiated samples subjected to a dose of $1 \mathrm{~Gy}$, which were found to be 4.5 and $2300 \mathrm{~ns}$ for the blue and orange RPL, respectively.

The following aspect of the acquisition of accurate and high-intensity 2D dose images using a disk-type Ag-activated detector system, as shown in Fig. 2(b), based on blue and orange RPL should be considered: in the case of the constant angular velocity (CAV) method used in this work, the linear velocity and transit time of the luminescence, moving across the spot size on the detector, are quite different at inner and outer positions on the rotating disk. When the lifetime is much shorter than the transit time, the luminescence will fade away rapidly and the accumulated RPL intensity will decrease; this is particularly relevant for the blue RPL, which has a shorter lifetime, on the order of a few nanoseconds. Therefore, when the disk is revolving at a constant rate of $2400 \mathrm{rpm}$, as in this work, the RPL intensity depends strongly on the radiative lifetime of the material and the spot size. For example, the transit time of the luminescence, moving across the spot size of nearly 1 $\mu \mathrm{m}$ in diameter focused by an objective lens $(100 \times$, numerical aperture $\mathrm{NA}=0.95)$, can be estimated to be as $80 \mathrm{~ns}$ at a radius of $\mathrm{r}=50 \mathrm{~mm}$ and $530 \mathrm{~ns}$ at $\mathrm{r}=7.5 \mathrm{~mm}$.

Fig. 4 (a) presents a photographic image of the mask used ("Hassam House" in Kobe). The detector was irradiated with soft X-rays passing through this stainless steel mask, which was $250 \mu \mathrm{m}$ thick and was attached to the detector. Figs. 4(b) and 4(c) present two reconstructed dose distributions obtained from the disk-type Ag-activated phosphate glass detector using the orange and blue RPL, respectively, for an absorbed dose of 3 Gy. The full-scale input voltage of the $\mathrm{A} / \mathrm{D}$ converter was set between 0 and $2.5 \mathrm{~V}$ with a resolution 
of 16 bits. As described above, in the case of the blue RPL, the intensity of the reconstructed dose distributions will be higher for a smaller spot size and/or faster rotation of the detector.

By adjusting the full-scale input voltage to lie between 0 and $0.1 \mathrm{~V}$ after the acquisition of the reconstructed 2D image presented in Fig. 4(b), lower-level distributions could be observed, as low as several mGy, as shown in Fig. 4(d). An enlarged map of a portion of the image is presented in Fig. 4(e). Thus, using an RPL detector, steep gradients in dose distribution levels can be clearly resolved with high image contrast.

Fig. 5 presents a series of $2 \mathrm{D}$ reconstructed dose distributions obtained using the disk-type Ag-activated glass detector, which was rotating at a rate of $2400 \mathrm{rpm}$, and the same mask as was previously used for X-ray irradiation at a dose of $3 \mathrm{~Gy}$. The use of a confocal detection system and a 0.90 NA objective lens with a working distance of $1 \mathrm{~mm}$ enabled the reconstruction of $3 \mathrm{D}$ dose distributions recorded within the transparent detector by combining images acquired at different depths, i.e., near the surface and 100, 200,300 and $400 \mu \mathrm{m}$ below the surface. Such as a $2 \mathrm{D}$ dose distribution acquired as a function of different depths within the sample are necessary to calibrate or compare the measured depth distribution of active luminescent centres with the deposited energy curves of X-ray radiation in the material. This approach is currently underway.

\section{Conclusions}

The data obtained in this study led to the following conclusions: 
(1) The steady- and transient-state optical properties of the blue and orange RPL of Ag-activated phosphate glass, including the time-resolved spectra and nanosecond-time-scale decay curves, were compared.

(2) Some advantages of using the blue RPL instead of the typically used orange RPL were demonstrated and discussed. Specifically, the use of the blue RPL allows for the high-speed acquisition of dose images.

(3) The ability to reconstruct $2 \mathrm{D}$ and $3 \mathrm{D}$ dose images with a high spatial resolution on the order of microns, a wide dynamic range spanning three orders of magnitude, and non-destructive readout was successfully demonstrated.

It should be possible for the three stages of operation of an Ag-activated phosphate glass detector — pre-heating, reading and erasing — to be performed optically, without the need to use a thermal heating oven, as is typically done; studies of this approach are currently underway. The investigated detector, based on the RPL phenomenon, exhibits superior characteristics and should be suitable for 2D and 3D dose imaging applications such as fluorescent nuclear track detection (G. M. Akselrod et al., 2006) and micro-radiography (G. Baldacchini et al., 2002).

\section{Acknowledgements}

T. K would like to thank Prof. H. Nanto at Kanazawa Institute of Technology for valuable discussions. This work was supported by JSPS, Grant-in-Aid for Scientific

Research (B), No. 26289362 and partially by JST A-step, No. AS251Z01722P and AS262Z00986P. 


\section{References}

Akselrod G. M., Akselrod, M. S., Benton, E. R., Yasuda, N., 2006. A novel $\mathrm{Al}_{2} \mathrm{O}_{3}$ fluorescent nuclear track detector for heavy charged particles and neutrons, Nucl. Instr. Meth. Phys. Res. B 247, 295-306.

Baldacchini, G., Bonfigli, F., Faenov, A., Flora, F., Montereali, R. M., Pace, A., Pikuz, T., Reale, L., 2003. Lithium fluoride as a novel X-ray image detector for biological $\mu$-world capture”, J. Nanosci. Nanotech. 3, 483-486.

Kurobori, T., Zheng, W., Miyamoto, Y., Nanto, H., Yamamoto, T., 2010. The role of silver in the radiophotoluminescent properties in silver-activated phosphate glass and sodium chloride crystal. Opt. Mater. 32, 1231-1236.

Kurobori, T., Nakamura, S., 2012. A novel disk-type X-ray area imaging detector using radiophotoluminescence in silver-activated phosphate glass. Radiat. Meas. 47, 1009-1013.

Kurobori, T., Miyamoto, Y., Maruyama, Y., Yamamoto, T., Sasaki, T., 2014a. A comparative study of optical and radiative characteristics of X-ray-induced luminescent defects in Ag-doped glass and LiF thin films and their applications in 2-D imaging, Nucl. Instr. Meth. Phys. Res. B. 326, 76-80.

Kurobori, T., Maruyama, Y., Miyamoto, Y., Sasaki, T., Yamamoto, T., 2014b. "Non-destructive readout of $2 \mathrm{D}$ and $3 \mathrm{D}$ dose distributions using a disk-type radiophotoluminescent glass plate", $12^{\text {th }}$ Europhysical Conference of Defects in Insulating Materials (Eurodin 2014), Canterbury, UK, poster No.1.

Montereali, R. M., Baldacchini, G., Martell, S., Scavarda do Carmo, L. C., 1991. LiF films: Production and characterization. Thin Solid Films 196, 75-83.

Perry, J. A., 1987. RPL Dosimetry, Radiophotoluminescence in Health Physics. Taylor \& Francis, New York, pp.21.

Yamamoto T., Maki D., Sato F., Miyamoto Y., Nanto H., Iida T., 2011. The recent investigations of radiophotoluminescence and its application. Radiat. Meas. 46, 1554-1559.

Yokota, R., Imagawa, H., 1966. Radiophotoluminescent centers in silver-activated phosphate glass. J. Phys. Soc. Jpn 23, 1038-1048.

Zheng, W., Kurobori, T., 2011. Assignments and optical properties of X-ray-induced colour centres in blue and orange radiophotoluminescent silver-activated glasses. J. Lumin. 131, 36-40. 


\section{FIGURE CAPTIONS:}

Fig. 1. Operating principles of the RPL glass detector system: the recording, reading and erasing processes.

Fig. 2. (a) Acquisition setup for the measurement of the time-resolved RPL spectra and decay curves of the X-ray-irradiated Ag-activated phosphate glass. A laser beam with a rectangular beam shape was incident on a side facet of the sample. (b) Schematic diagram of a disk-type RPL glass detector and a confocal optical detection system.

Fig. 3. (a) RPL spectra excited at $340 \mathrm{~nm}$ before and after X-ray irradiation. (b) Time-resolved RPL spectra for a UV laser at $349 \mathrm{~nm}$ after several pulse delays. (c) Luminescent decay curves acquired at 450 and $650 \mathrm{~nm}$ for the blue and orange RPL, respectively.

Fig. 4 (a) Photographic image of the mask used for X-ray irradiation. Also presented are two reconstructed dose distributions acquired with the full-scale input voltage set between 0 and $2.5 \mathrm{~V}$ using the (b) orange and (c) blue RPL, (d) an image acquired with the full-scale input voltage set between 0 and $0.1 \mathrm{~V}$, and (e) an enlarged portion of the image presented in (b).

Fig. 5 Reconstructed dose distributions for images recorded at different depths using the orange RPL. 


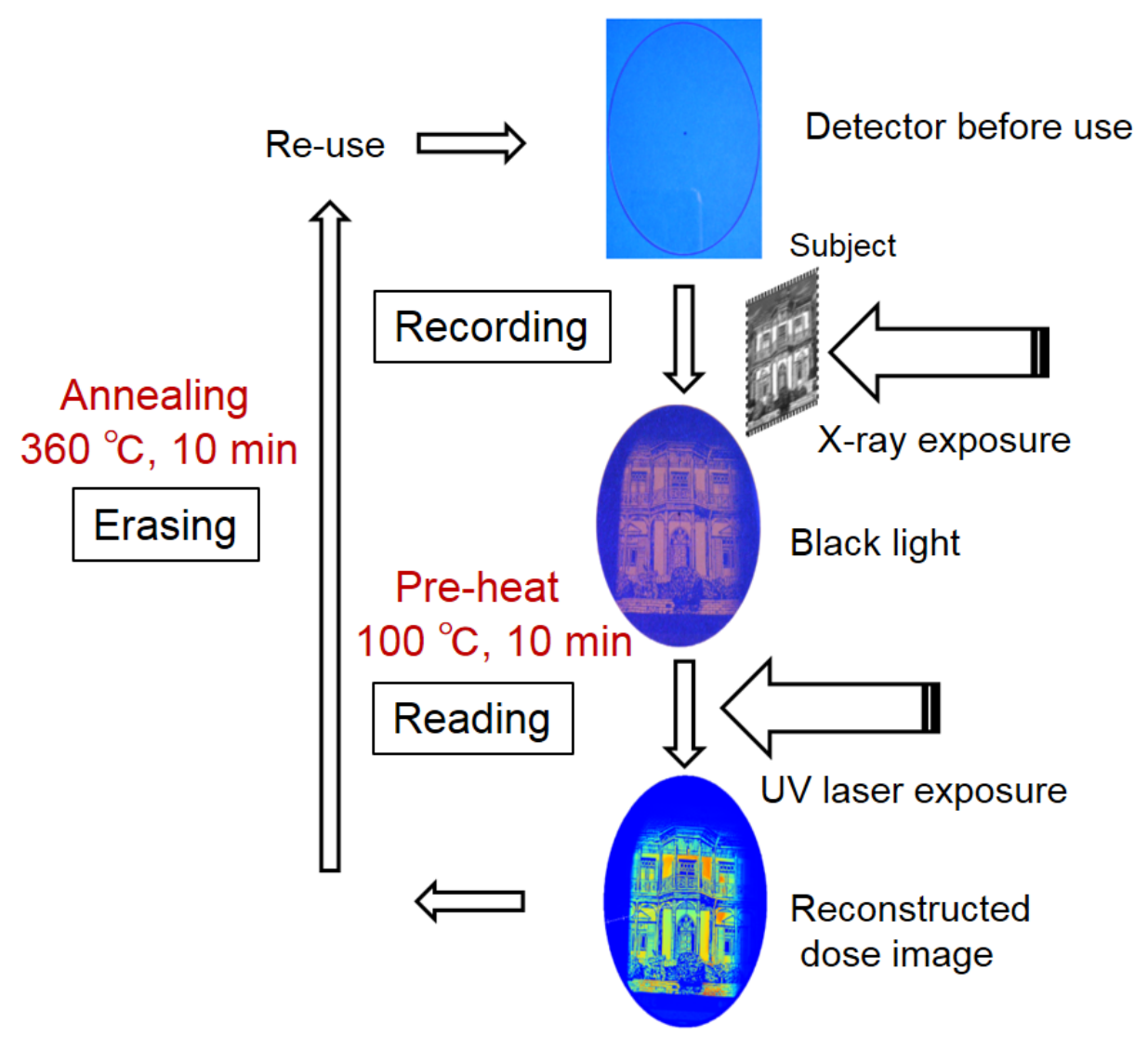

1-column Fig. 1 T. Kurobori 

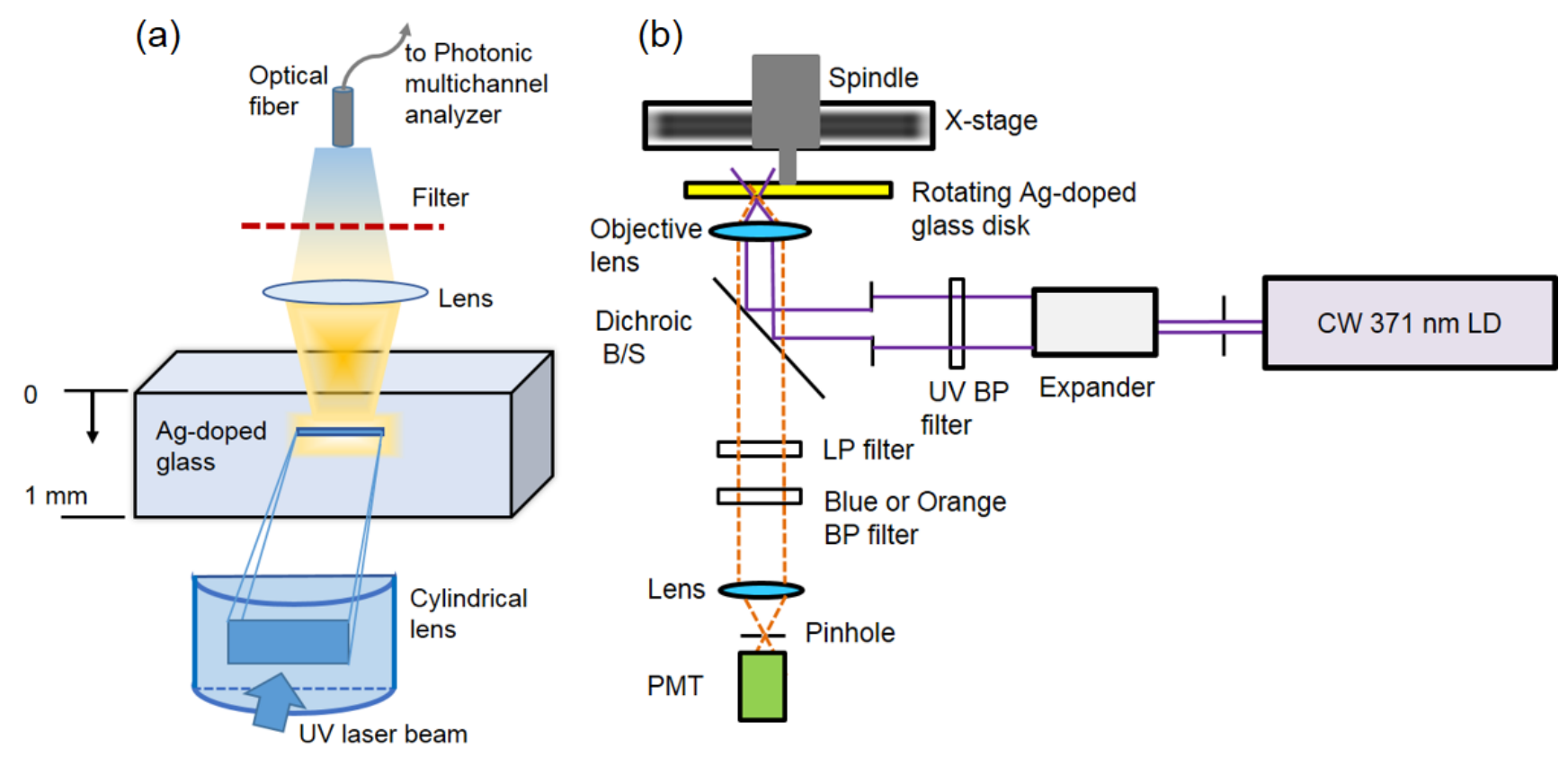

\section{5-column Fig. 2 T. Kurobori}


(a)

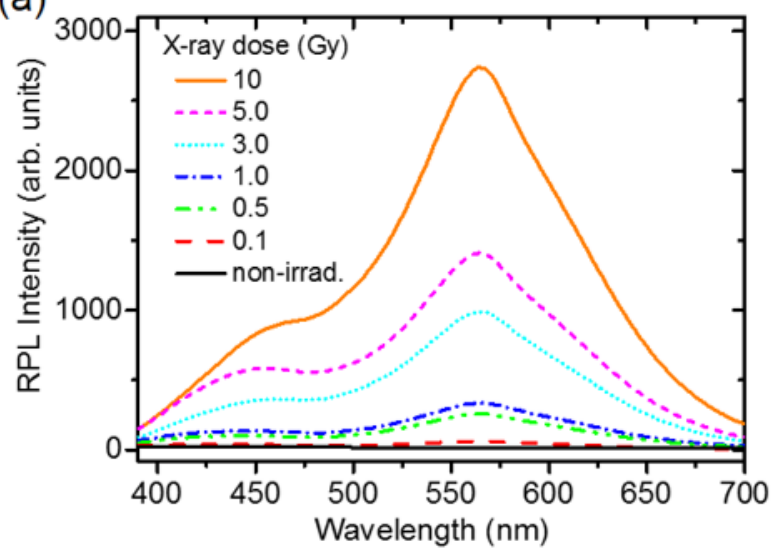

(b)

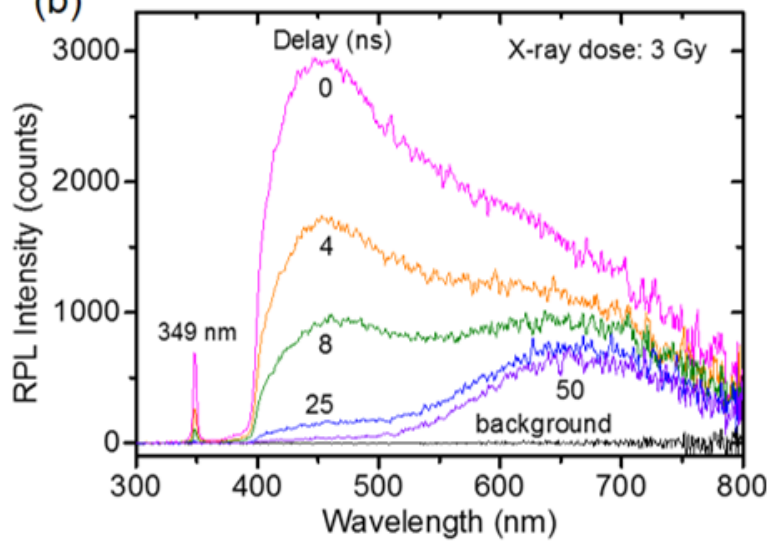

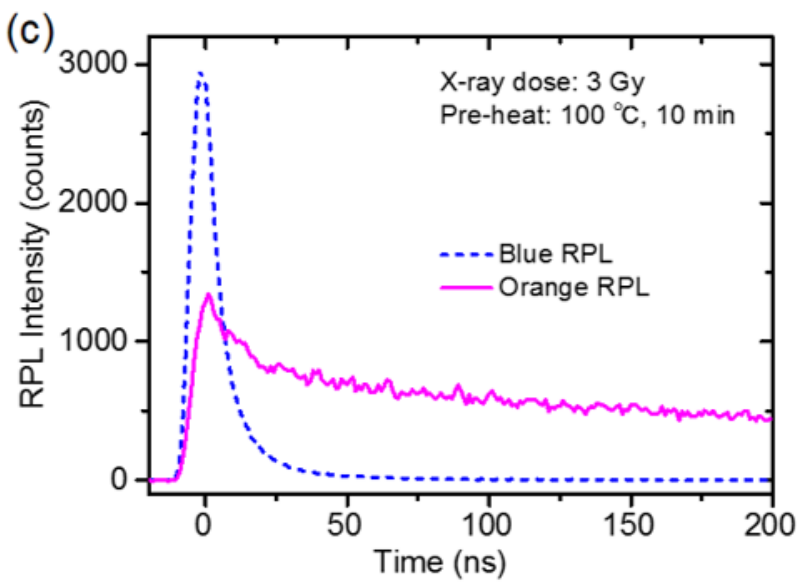

\section{5-column Fig. 3 T. Kurobori}



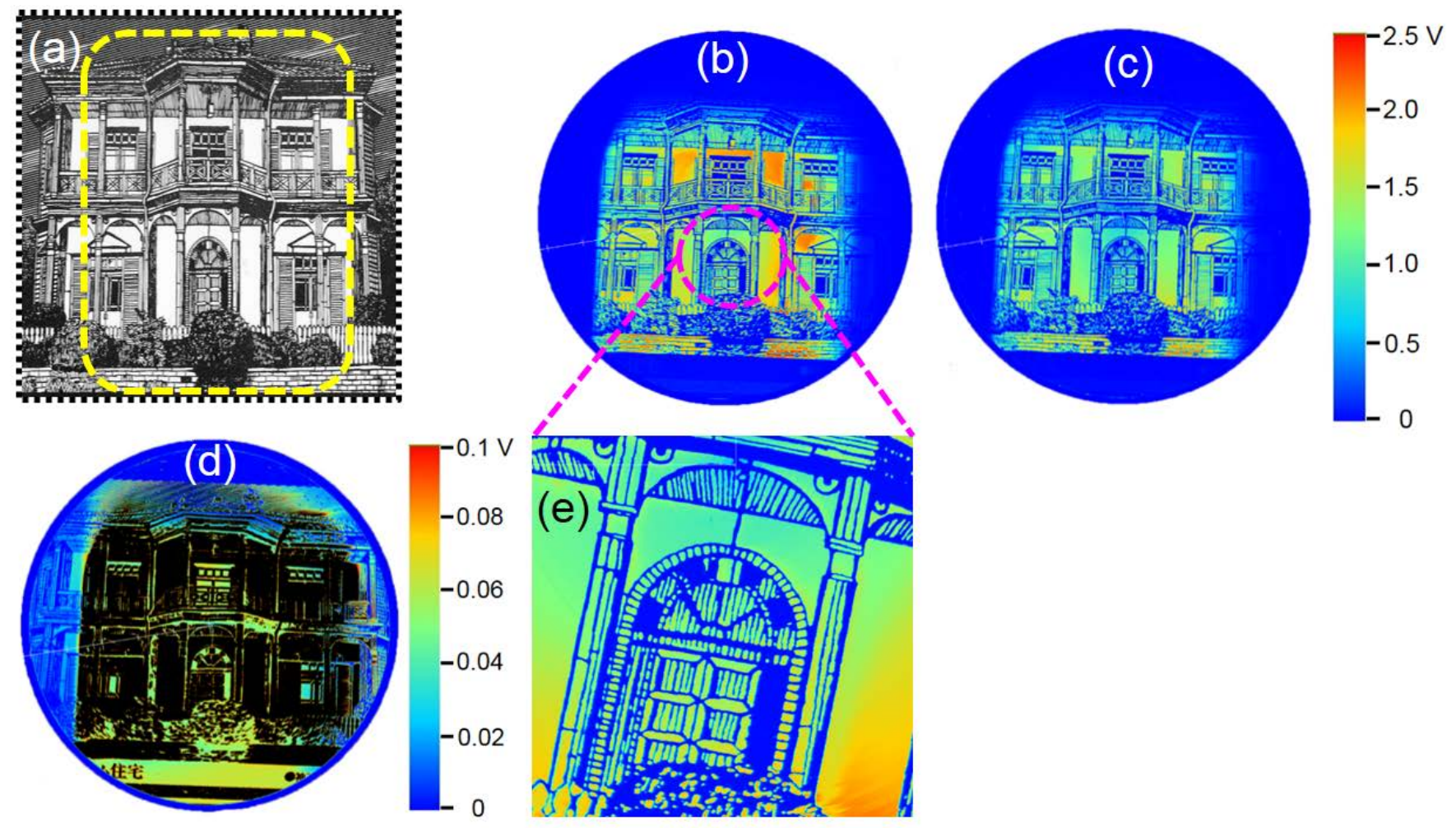

1.5-column Fig. 4 T. Kurobori 


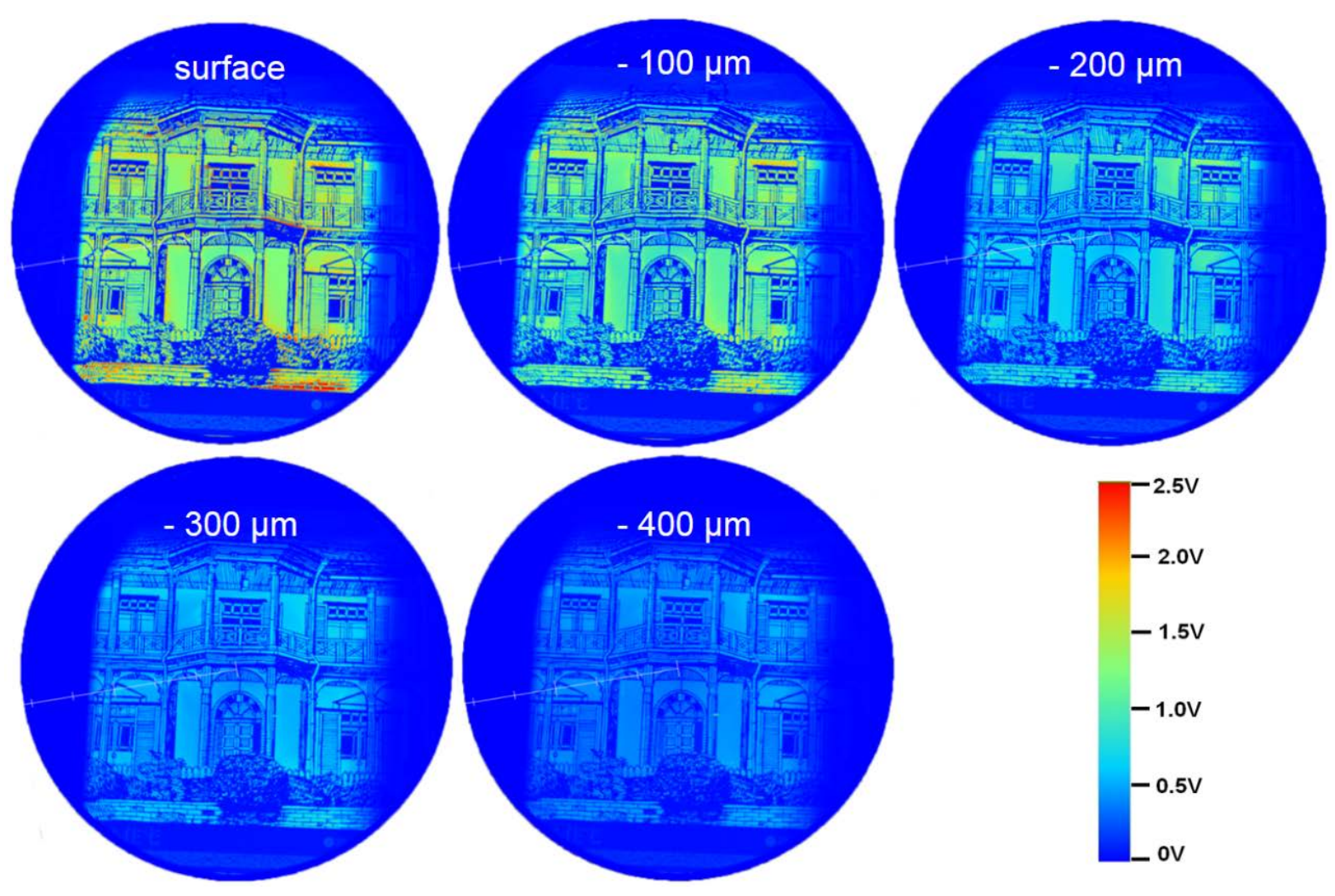

1.5-column Fig. 5 T. Kurobori 